\title{
Comparing turbulent parameters obtained from LITOS and radiosonde measurements
}

\author{
A. Schneider, M. Gerding, and F.-J. Lübken \\ Leibniz Institute of Atmospheric Physics at the University of Rostock, Kühlungsborn, Germany \\ Correspondence to: A. Schneider (schneider@iap-kborn.de)
}

Received: 28 May 2014 - Published in Atmos. Chem. Phys. Discuss.: 22 July 2014

Revised: 24 December 2014 - Accepted: 26 January 2015 - Published: 27 February 2015

\begin{abstract}
Stratospheric turbulence is important for the mixing of trace species and the energy balance, but direct measurements are sparse due to the required resolution and accuracy. Recently, turbulence parameters such as the energy dissipation rate $\varepsilon$ were inferred from standard radiosonde data by means of a Thorpe analysis. To this end, layers with vertically decreasing potential temperature are analysed, which is expected to indicate turbulence. Such an application assumes a proportionality between the Thorpe length $L_{\mathrm{T}}$ and the Ozmidov scale $L_{\mathrm{O}}$. While this relation is accepted for the ocean, experimental evidence for such proportionality in the stratosphere is sparse. We have developed a high-resolution $(8 \mathrm{kHz})$ turbulence measurement system called LITOS (Leibniz Institute Turbulence Observations in the Stratosphere), which for the first time resolves the inner scale of turbulence in the stratosphere. Therewith the energy dissipation rate $\varepsilon$ can be determined by spectral analysis. This independent value for $\varepsilon$ enables us to check the relation $L_{\mathrm{O}} \propto L_{\mathrm{T}}$. In our measurements no such proportionality can be seen, although the mean of the ratio $L_{\mathrm{O}} / L_{\mathrm{T}}$ is close to what is assumed in radiosonde analyses. Dissipation rates for individual layers obtained from radiosondes deviate up to a factor of $\sim 3000$ from those obtained by spectral analysis. Some turbulent layers measured by LITOS are not observed by the radiosonde at all, and vice versa. However, statements about the statistical mean seem to be possible by Thorpe analysis.
\end{abstract}

\section{Introduction}

Although the stratosphere is mostly stably stratified, breaking of gravity waves and instabilities cause turbulence and energy dissipation. This modifies the energy transport from the troposphere to the mesosphere. The amount of energy converted into heat is described by the turbulent energy dissipation rate $\varepsilon$. Moreover, turbulence is an important parameter for the vertical mixing of trace species. As in the stratosphere turbulent dissipation occurs on small scales of centimetres and below, measurements are technically challenging and therefore sparse (e.g. Barat, 1982; Theuerkauf et al., 2011).

In order to enlarge the knowledge of turbulence by exploiting existing measurements available for large geographical areas and several years, the extraction of turbulence parameters such as $\varepsilon$ from standard radiosonde data (vertical resolution $5 \mathrm{~m}$ ) has been proposed (Clayson and Kantha, 2008). The evaluation uses the method developed by Thorpe (1977, 2005) to detect static instabilities as a proxy for turbulence. Note that such a measurement is somewhat different from measuring the turbulent motions directly, as done by LITOS. For example, within an instability turbulence may have not yet been developed or, on the other hand, turbulence might be still active while the instability has already deceased. Additionally, turbulence may not be related to static instabilities at all.

The Thorpe analysis of unstable layers is done by comparing a measured potential temperature profile to an equivalent (statically) stable one obtained by sorting. This means that the order of the data points is changed upwards and downwards to yield a statically stable profile with monotonously increasing potential temperature. Precisely, the Thorpe displacement $D_{\mathrm{T}}$ is defined by the vertical displacements needed for the sorting; i.e. if an air parcel at altitude $z_{j}$ is sorted to $z_{k}$, then the Thorpe displacement at $z_{j}$ is $D_{\mathrm{T}}\left(z_{j}\right)=$ $z_{j}-z_{k}$. The Thorpe length is the root mean square of the 
Thorpe displacements taken over an unstable layer:

$L_{\mathrm{T}}=\operatorname{rms}\left(D_{\mathrm{T}}\right)$.

It describes the distance over which heavier air parcels are carried above lighter ones. Wilson et al. $(2011,2010)$ use the Thorpe method for statistical analysis without computing dissipation rates.

The Ozmidov length scale

$L_{\mathrm{O}}=\alpha \sqrt{\frac{\varepsilon}{N^{3}}}$,

where $\varepsilon$ is the (kinetic) energy dissipation rate, $N$ the BruntVäisälä frequency and $\alpha$ a numerical constant near unity, represents the vertical scales of the largest turbulent eddies (Ozmidov, 1965). For the determination of the dissipation rate from a Thorpe analysis, the key assumption is a proportionality between Thorpe and Ozmidov lengths, $L_{\mathrm{O}} \propto L_{\mathrm{T}}$. This relation has been extensively studied in the ocean, and the assumption is fulfilled to a good extent (Thorpe, 2005; Dillon, 1982; Wesson and Gregg, 1994). But for the atmosphere there are only few examinations of the proportionality (e. g. Gavrilov et al., 2005; Kantha and Hocking, 2011; Wilson et al., 2014). With our new high-resolved instrument LITOS (Leibniz Institute Turbulence Observations in the Stratosphere) (Theuerkauf et al., 2011), the energy dissipation rate $\varepsilon$ is obtained independent of $L_{\mathrm{T}}$ by means of spectral analysis of wind fluctuations. Thus it is possible to check the relation $L_{\mathrm{O}} \propto L_{\mathrm{T}}$.

Please note that our comparison involves two parameters: (a) evaluation method (Thorpe or spectral analysis) and (b) vertical resolution (low or high). We concentrate on results from high-resolved spectral analysis (as a very precise method of $\varepsilon$ determination) and low-resolved Thorpe analysis. Such a Thorpe evaluation of radiosonde data has been proposed for extensive use (Clayson and Kantha, 2008; Love and Geller, 2012). Note that Love and Geller (2012) call $1 \mathrm{~Hz}$ $(5 \mathrm{~m})$ high resolution, while we call it low resolution (compared to LITOS with $8 \mathrm{kHz}$ ). In principle, the Thorpe analysis can also be performed on data with higher resolution, as done, e.g., by Luce et al. (2002) for temperature data with a $50 \mathrm{~Hz}$ sampling rate; however, these data are rarely available compared to those of standard radiosonde. Furthermore, a kind of spectral analysis can be used to determine dissipation rates from low-resolution wind data (Barat, 1982), but this method depends on the absolute value of the wind velocity, which is not available for our measurements (see next section).

In Sect. 2, the measurement principle of LITOS and the determination of the energy dissipation rates with both methods are shortly reviewed. The independent measurements of $L_{\mathrm{O}}$ and $L_{\mathrm{T}}$ are compared in Sect. 3. Section 4 shows results for the energy dissipation rate $\varepsilon$ from both a Thorpe analysis and our high-resolved spectral analysis. Conclusions are drawn in Sect. 5.

\section{Instrumentation and methods}

As described in Theuerkauf et al. (2011), LITOS is a balloonborne instrument which measures winds with high vertical resolution of millimetres. The wind sensor is a constant temperature anemometer (CTA), which facilitates the cooling effect on a heated wire of $5 \mu \mathrm{m}$ diameter. To infer wind velocities from the anemometer voltage, a calibration in the same ambient conditions (pressure, temperature) is required. This is not possible for a balloon flight, as the pressure varies within several orders of magnitude during the flight. Nevertheless, we are only interested in the spectral form, and the absolute values are not important (see below); therefore we use the anemometer voltage for the analysis. The vertical resolution is obtained by applying a sample rate of $8 \mathrm{kHz}$ with a balloon ascent rate of $5 \mathrm{~m} \mathrm{~s}^{-1}$. To date, three flights on large $\left(\sim 10000 \mathrm{~m}^{3}\right)$ balloons have been performed, namely Balloon Experiments for University Students (BEXUS) 6, 8 and 12 in 2008, 2009 and 2011, respectively. They were launched at Kiruna $\left(68^{\circ} \mathrm{N}, 21^{\circ} \mathrm{E}\right)$ in autumn. For BEXUS 6, the radiosonde data are partly disturbed so that it is not considered in this article.

The left panel in Fig. 1 shows an example of a time series of the anemometer voltage of the BEXUS 12 flight. Large-scale motions have already been removed by subtracting a spline. At altitudes with small variations $(\lesssim 1 \mathrm{mV}$, e.g. from 10.28 to $10.3 \mathrm{~km}$ ), the signal mainly shows instrumental noise; this corresponds to a calm region. Large fluctuations, as in the height range of 10.18 to $10.28 \mathrm{~km}$, correspond to turbulence. Note that there is a substructure which divides the turbulent region into different patches. For the patch from 10.27 to $10.28 \mathrm{~km}$ (shaded in the graph), the power spectral density (PSD) is plotted in the right panel of Fig. 1 (blue curve). An inertial regime with a $-5 / 3$ slope and the transition to the viscous subrange with a -7 slope is identified. The part below $\sim 10^{-2} \mathrm{~m}$ spatial scale with approximately constant PSD corresponds to the instrumental noise level. As the transition to the inertial range is resolved, a fit of the Heisenberg (1948) model in the form given by Lübken (1992) is applied to the experimental data (red curve). This gives the inner scale $l_{0}$, i.e. the transition from the inertial to the viscous subrange. In the example, $l_{0}=1.9 \times 10^{-2} \mathrm{~m} \pm 4.5 \times 10^{-3} \mathrm{~m}$ (fit error). Note that $l_{0}$ does not depend on the absolute value of the PSD, only on identifying the bend in the spectrum. From the inner scale, the energy dissipation rate is obtained by

$\varepsilon=c^{4} \frac{v^{3}}{l_{0}^{4}}$,

where $v$ is the kinematic viscosity (derived from the radiosonde measurement of temperature and pressure on the same gondola), and $c=5.7$ (Theuerkauf et al., 2011; Haack et al., 2014). For the example in Fig. 1, $\varepsilon=3.2 \times$ $10^{-4} \mathrm{~W} \mathrm{~kg}^{-1} \pm 3.0 \times 10^{-4} \mathrm{~W} \mathrm{~kg}^{-1}$. 

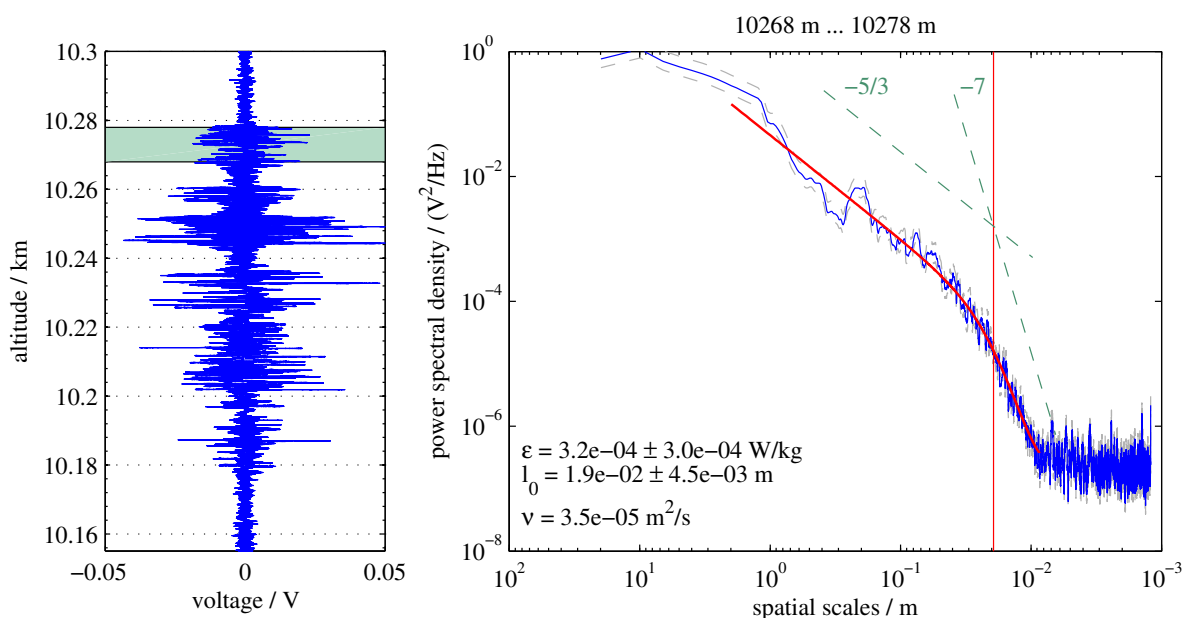

Figure 1. Example of raw data (left) and associated power spectrum (right) computed for the shaded area in the raw data plot. In the raw data, an amplitude of $\lesssim 1 \mathrm{mV}$ corresponds to instrumental noise. In the spectrum, the blue curve shows the measurement, the grey dashed lines the $95 \%$ confidence interval and the red curve the fit of the Heisenberg model to the measured spectrum; the red vertical line indicates the inner scale $l_{0}$. The green dashed lines display slopes of $-5 / 3$ and -7 . The errors given are fit errors.

In order to obtain a vertical profile of energy dissipation rate, a sliding window of $5 \mathrm{~s}$ (roughly $25 \mathrm{~m}$ altitude) is used. For each window, $\varepsilon$ is computed according to the procedure described above. For non-turbulent spectra, $\varepsilon$ is set to 0 . A spectrum is regarded as non-turbulent if the noiselevel detection fails, if the inner scale $l_{0}$ is not within the fit range, if $\varepsilon$ has implausible values (less than 0 or greater than $100 \mathrm{~W} \mathrm{~kg}^{-1}$ ) or if the mean distance between the fit and the data is larger than a fixed threshold. That means the decision is made automatically based on a set of objective criteria. The resulting $\varepsilon$ profile has a vertical resolution of $\sim 10 \mathrm{~m}$ due to the selected overlap.

The Thorpe analysis is performed similar to the procedure described in Wilson et al. (2011) on data from a Vaisala RS92 radiosonde, which was on the same gondola as the CTA sensors. Moisture is handled using the routine given by Wilson et al. (2013). To this end, saturated regions are detected, and a composite potential temperature profile $\Theta_{*}$ is computed by integration of $\partial \Theta / \partial z$ using the moist buoyancy frequency within those saturated regions and the dry buoyancy frequency otherwise. The left panel of Fig. 2 shows the potential temperature profile for the BEXUS 12 flight. In the inset, the part from 15.48 to $15.80 \mathrm{~km}$ is magnified for better visibility of instabilities, which manifest as negative gradients of potential temperature. The Thorpe displacement is shown in the right panel of Fig. 2. Large displacements correspond to large vertical extents of unstable layers. To identify unstable layers and their vertical extension, the cumulative sum of the Thorpe displacement (which is negative within an unstable region and 0 within a stable one) is used. To select real overturns and discard negative potential temperature gradients originating from measurement noise, a statistical test is applied. To this end, the range of the potential temper-
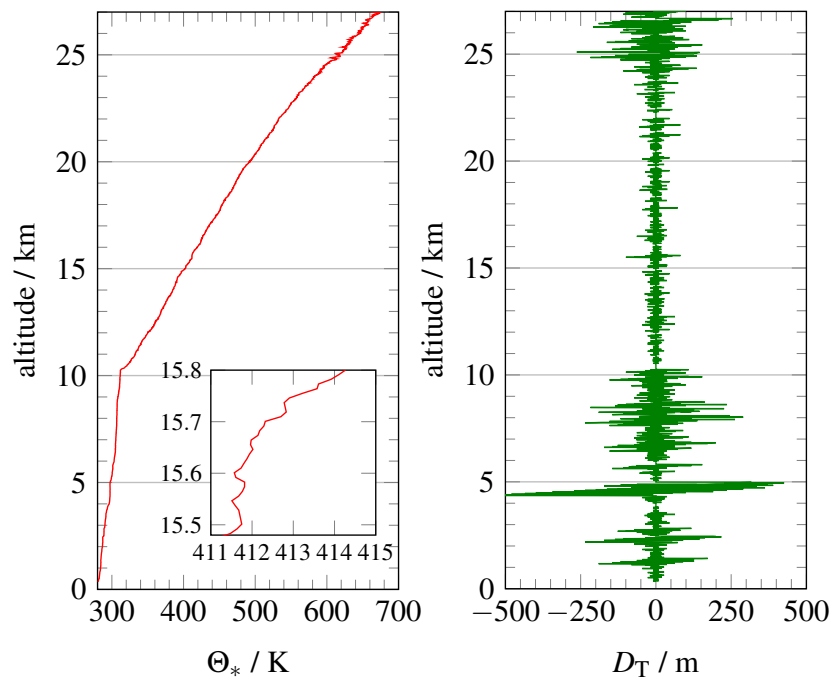

Figure 2. Potential temperature profile (left) and Thorpe displacement (right) for the BEXUS 12 flight. The inset in the left panel shows a magnification from 15.48 to $15.80 \mathrm{~km}$ for better visibility of instabilities (manifested as negative gradients of potential temperature).

atures within an inversion is compared with the range of a pure noise sample of the same length (Wilson et al., 2010). We assume here that the standard deviation of instrumental noise in segments of $200 \mathrm{~m}$ is described by half the square of the standard deviation of first differences of the potential temperature after trend removal. For each detected unstable layer, the Thorpe length is computed according to Eq. (1).

Only significant overturns with a $99 \%$ percentile are used, discarding $\sim 45 \%$ (BEXUS 8 ) and $\sim 30 \%$ (BEXUS 12) of the inversions as noise-induced. The mean trend-to-noise ra- 

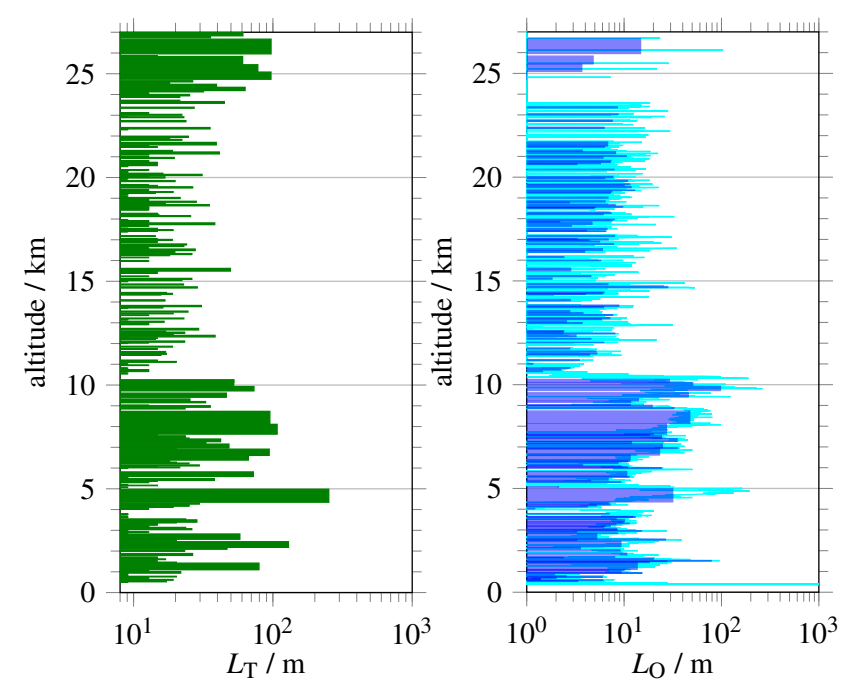

Figure 3. Thorpe length (left) and Ozmidov scale (right) vs. altitude for the detected inversions of the BEXUS 12 flight. In the right panel, the cyan curve shows the Ozmidov scale $L_{\mathrm{O}}$ in the full resolution of the LITOS profile, the blue bars visualise averages over the inversions detected by the radiosonde $\left(\overline{L_{\mathrm{O}}}\right)$.

tio (TNR) is $\bar{\xi}=1.7$ for the BEXUS 8 flight and $\bar{\xi}=4.1$ for the BEXUS 12 flight.

Several thin layers of only $10 \mathrm{~m}$ or $20 \mathrm{~m}$ passed the significance test. We are aware that this is on the edge of radiosonde capability. These thin layers would to a large extent be suppressed if the instrument noise were set to the standard deviation of first differences divided by $\sqrt{2}$ as done by Wilson et al. (2011). However, LITOS also shows many thin layers. Hence, this procedure would result in many fewer coincident layers especially in the stratosphere and, by this, bias the comparison. Nevertheless, as we will describe below, our main results are independent from the procedure of noise calculation.

\section{Comparison of Thorpe and Ozmidov scales}

A plot of the Thorpe length for the BEXUS 12 flight is shown in the left panel of Fig. 3. Unstable layers take up $50 \%$ of the altitude and can be found in the whole range. Large Thorpe lengths stand out, e.g., at $5 \mathrm{~km}$, between 5 and $10 \mathrm{~km}$ and near $25 \mathrm{~km}$ altitude, corresponding to the large values of $D_{\mathrm{T}}$ in the right panel of Fig. 2. Mean values of $L_{\mathrm{T}}$ are $29 \mathrm{~m}$ in the troposphere and $22 \mathrm{~m}$ in the stratosphere; i.e. the Thorpe length is slightly larger in the less stable troposphere.

The Ozmidov scale is computed from the energy dissipation rate obtained by LITOS, using Eq. (2) and $\alpha=1$. The Brunt-Väisälä frequency $N$ is calculated from the radiosonde data as it only slowly varies with altitude. In that computation, the sorted potential temperature profile is used instead of the original data, because a background stratification is needed and an imaginary $N$ should be avoided (Dil-

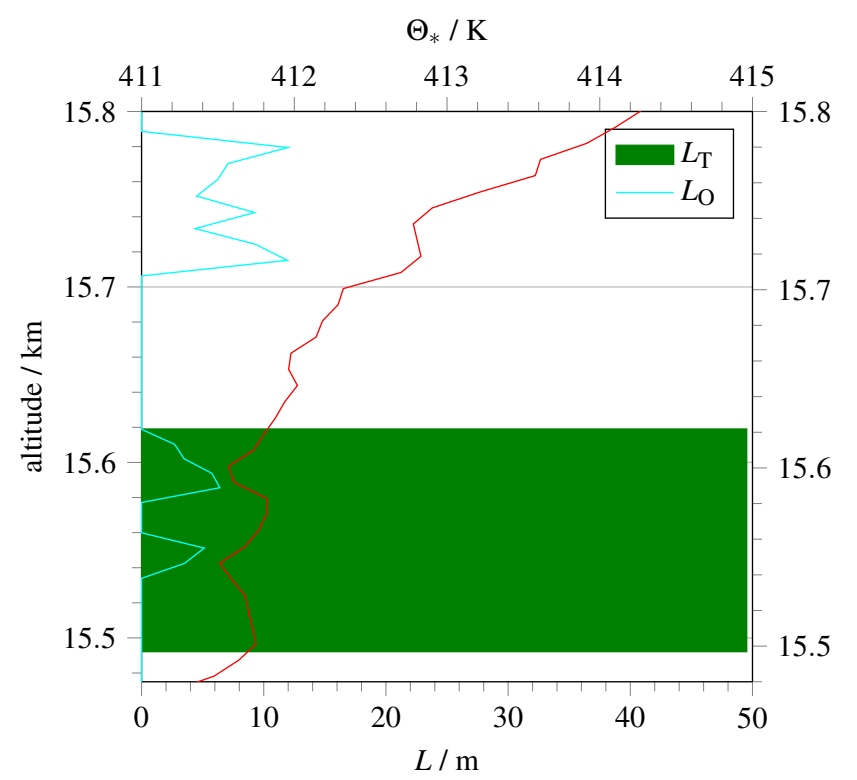

Figure 4. Zoomed plot of Thorpe (green) and Ozmidov (cyan) scales for the BEXUS 12 flight. The potential temperature is plotted in red.

lon, 1982, Sect. 3). The result for the BEXUS 12 flight is plotted in the right panel of Fig. 3 (cyan curve). According to LITOS, $53 \%$ of the atmosphere is turbulent, i.e. $\varepsilon>0$ and hence $L_{\mathrm{O}}>0$.

Figure 4 shows the Thorpe length and the Ozmidov scale for the altitude range of 15.48 to $15.80 \mathrm{~km}$. As LITOS computes $\varepsilon$ on a constant grid independent of the layers, the substructure of larger turbulent layers can be seen (e.g. from $\sim 15.5$ to $15.62 \mathrm{~km}$ ), while the Thorpe length is a perlayer value by construction. From 15.706 to $15.789 \mathrm{~km}$, only LITOS observes turbulence while the Thorpe method does not. The decrease of potential temperature is not significant, so the Thorpe method is blind for the turbulent motions.

In order to do a comparison between both length scales, the layers where both methods detect turbulence are selected. For BEXUS 8 (BEXUS 12), $86 \%$ (69\%) of the significant, unstable layers are also detected by LITOS, and $90 \%(88 \%)$ of the layers detected by LITOS intersect with a significant, unstable layer. The energy dissipation rate (obtained from LITOS) is averaged over the layer (as detected by the Thorpe analysis of radiosonde data). Such mean values over a Thorpe layer will be denoted by averaging brackets $\langle\cdot\rangle$. For each unstable layer, the resulting $\langle\varepsilon\rangle$ is plugged into Eq. (2) to infer an Ozmidov scale $\overline{L_{\mathrm{O}}}=\sqrt{\langle\varepsilon\rangle /\langle N\rangle^{3}}$ for the layer. The blue bar plot in the right panel of Fig. 3 shows a graph for the BEXUS 12 flight. The layer near $5 \mathrm{~km}$ with large $L_{\mathrm{T}}$, e.g., is also seen in $\overline{L_{\mathrm{O}}}$, albeit less pronounced. In contrast, at $\sim 10 \mathrm{~km}$ altitude the Ozmidov scale is larger than the Thorpe scale. Mean values of $\overline{L_{\mathrm{O}}}$ are $15 \mathrm{~m}$ in the troposphere and $6 \mathrm{~m}$ (i.e. only half the value) in the stratosphere. Thus, in qualitative agreement with Eq. (2), the less stable troposphere shows 


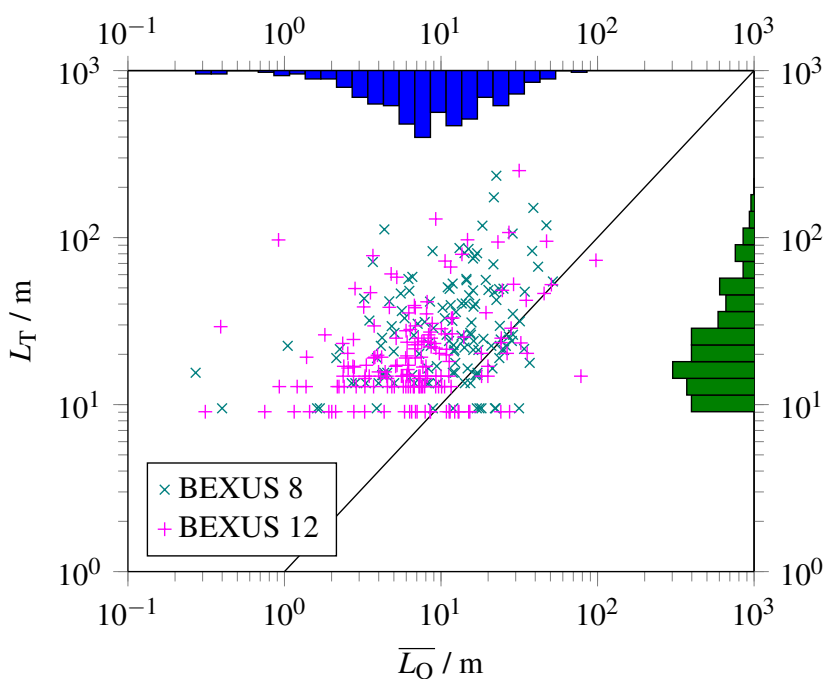

Figure 5. Thorpe scale $L_{\mathrm{T}}$ vs. Ozmidov scale $\overline{L_{\mathrm{O}}}$ for the BEXUS 8 (green) and 12 (magenta) flights. The black diagonal line represents $L_{\mathrm{O}}=L_{\mathrm{T}}$. The histograms show the distributions of $L_{\mathrm{O}}$ and $L_{\mathrm{T}}$, respectively, of the composite data set of BEXUS 8 and BEXUS 12, i.e. of all data points in the graph. The occurrence axes have a linear scale and are omitted for readability. Note that $L_{\mathrm{T}}$ is limited by the resolution of the radiosonde $(\sim 10 \mathrm{~m})$.

on average a larger Ozmidov scale, i.e. larger eddies. As the Thorpe length shows similar behaviour (see above), this generally supports the assumption of a relation between $L_{\mathrm{T}}$ and $L_{\mathrm{O}}$.

In Fig. 5 Ozmidov scale $\overline{L_{\mathrm{O}}}$ and Thorpe length $L_{\mathrm{T}}$ are plotted against each other for those 136 (175) significant, unstable layers of the BEXUS 8 (BEXUS 12) flight where turbulence has been detected by LITOS. Both length scales are of the same order of magnitude, but no direct relation between them can be seen in either flight. The correlation coefficient between both is 0.32 for BEXUS 8 and 0.33 for BEXUS 12 . Note that $L_{\mathrm{T}}$ is limited by the resolution of the radiosonde $(\sim 10 \mathrm{~m})$. An analogous plot for the simultaneous layers obtained using the more restrictive noise estimation of Wilson et al. (2011) contains similar scatter with no apparent correlation.

The histograms in the top and in the right axes in Fig. 5 show the distributions for $\overline{L_{\mathrm{O}}}$ and $L_{\mathrm{T}}$, respectively, for the composite dataset of BEXUS 8 and BEXUS 12. The maximum for the Thorpe length is slightly larger than for the Ozmidov scale. The decrease towards large scales is similar for both lengths. At small scales $L_{\mathrm{T}}$ is limited by the resolution of the radiosonde which produces the cut-off at $10 \mathrm{~m}$, while the histogram for $\overline{L_{\mathrm{O}}}$ shows a continuous decrease.

In contrast to our measurements, an approximate proportionality between Thorpe and Ozmidov lengths is observed in the ocean (e.g. Dillon, 1982; Wesson and Gregg, 1994; Thorpe, 2005). For example, Wesson and Gregg (1994) find that most of their data fall between $L_{\mathrm{O}}=4 L_{\mathrm{T}}$ and $L_{\mathrm{O}}=$

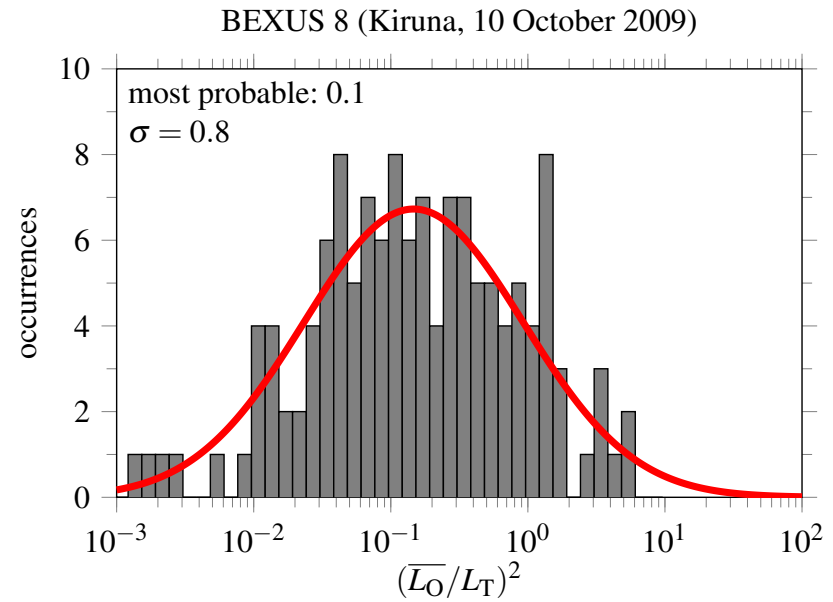

BEXUS 12 (Kiruna, 27 September 2011)

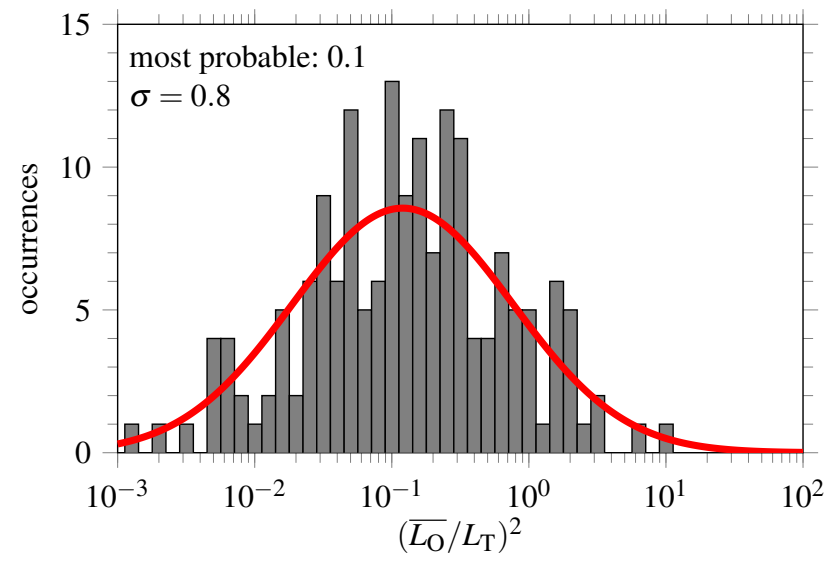

Figure 6. Statistics for the ratio $\left(\overline{L_{\mathrm{O}}} / L_{\mathrm{T}}\right)^{2}$ for the BEXUS 8 (top) and BEXUS 12 (bottom) flights. The red curves show the most likely normal distributions for the logarithmic data.

$(1 / 4) L_{\mathrm{T}}$, with a range from $10^{-2} \mathrm{~m}$ to $10^{2} \mathrm{~m}$. This is the basis for applying the Thorpe analysis on atmospheric data. Several authors (e.g. Gavrilov et al., 2005; Clayson and Kantha, 2008; Kantha and Hocking, 2011) have inferred energy dissipation rates from the Thorpe analysis by plugging $L_{\mathrm{O}}=c L_{\mathrm{T}}$ into Eq. (2) and solving for $\varepsilon$, thus getting

$\varepsilon=c^{2} L_{\mathrm{T}}^{2} N^{3}$.

Knowledge about the constant $c^{2}$ is very limited (see discussion below), and only Gavrilov et al. (2005) provide some information based on stratospheric data. With our high-resolved wind data we can determine this constant independently. Wijesekera et al. (1993) found the distribution of the ratio $L_{\mathrm{T}} / L_{\mathrm{O}}$ to be lognormal, which implies $\left(L_{\mathrm{O}} / L_{\mathrm{T}}\right)^{2}=c^{2}$ to be lognormal as well. Logarithmic histograms of $\left(\overline{L_{\mathrm{O}}} / L_{\mathrm{T}}\right)^{2}$ for the BEXUS 8 and BEXUS 12 flights are presented in Fig. 6. The red curves display the most likely normal distributions for the logarithmic data. They show a sufficient agreement to the histograms and are both centred around $\sim 0.1$. The distribution of values is fairly 

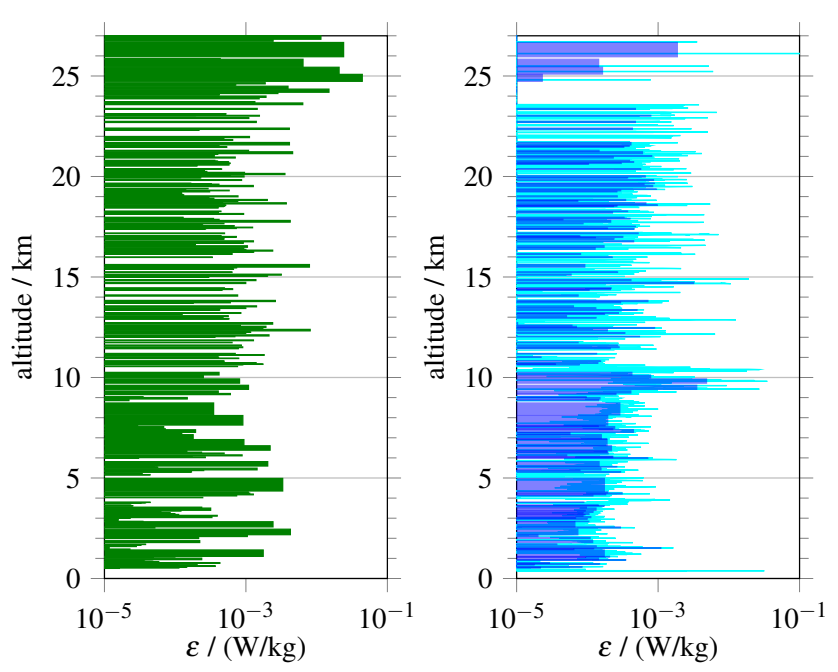

Figure 7. Energy dissipation rates from Thorpe analysis of the radiosonde (left) and spectral analysis of the high-resolved wind measurement (right) for the BEXUS 12 flight. In the right panel, the cyan curve shows $\varepsilon$ in the full resolution, the blue bars visualise averages over the unstable layers detected by the Thorpe analysis $(\langle\varepsilon\rangle)$.

broad: the full width at half maximum (FWHM) spans $\sim 1.9$ orders of magnitude.

We have calculated the same histograms also for the layers that are extracted using the more restrictive noise estimation of Wilson et al. (2011). Even if here only 36 (15) coincident layers are detected for BEXUS 8 (BEXUS 12), the distribution of $\left(L_{\mathrm{O}} / L_{\mathrm{T}}\right)^{2}$ is of similar width. The most probable values are slightly lower but agree within the uncertainty given by the distribution of the data.

\section{Energy dissipation rates}

The relation between the energy dissipation rate $\varepsilon$ and the length scales discussed above involves the Brunt-Väisälä frequency; see Eqs. (2) and (4). Thus the $\varepsilon$ values computed from LITOS via spectral analysis and the ones from the radiosonde via Thorpe analysis have to be compared separately.

The left panel of Fig. 7 shows an altitude profile of energy dissipation rates obtained from the Thorpe analysis of the radiosonde on BEXUS 12, assuming $L_{\mathrm{O}}=c L_{\mathrm{T}}$ with $c^{2}=0.3$ as in Clayson and Kantha (2008). In the right panel, the altitude profile of energy dissipation obtained from LITOS is plotted in cyan, while the blue bars depict the mean values $\langle\varepsilon\rangle$ over the unstable layers detected by the Thorpe analysis, for comparability. On average, the values are of the same order of magnitude, and the profiles have a similar structure. The large dissipation near $\sim 10 \mathrm{~km}$ in the CTA data does not stand out in the Thorpe analysis. The mean value over all significant, unstable layers from Thorpe, $1 \mathrm{~mW} \mathrm{~kg}^{-1}$, is larger

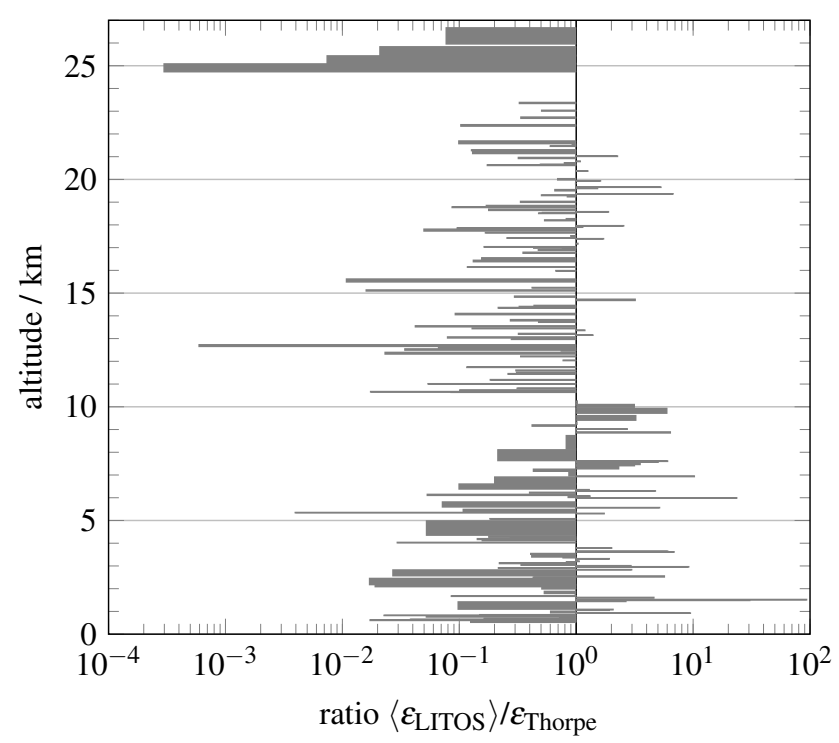

Figure 8. Ratio between energy dissipation rates from spectral analysis and from Thorpe analysis for the significant, unstable layers of the BEXUS 12 flight.

than the one from LITOS, $0.3 \mathrm{~mW} \mathrm{~kg}^{-1}$. For BEXUS 8 the averages are $3 \mathrm{~mW} \mathrm{~kg}^{-1}$ from Thorpe and $2 \mathrm{~mW} \mathrm{~kg}^{-1}$ from LITOS. That fits the fact that the used value for $c^{2}, 0.3$, is larger than the one obtained from our own data, $c^{2}=0.1$ (cf. Fig. 6). If the whole $\varepsilon$ profile (not only the unstable layers detected by Thorpe) is taken into account, the average dissipation rate obtained by LITOS is $0.4 \mathrm{~mW} \mathrm{~kg}^{-1}$ for BEXUS 12 and $2 \mathrm{~mW} \mathrm{~kg}^{-1}$ for BEXUS 8 . To get a closer look, the deviation of $\langle\varepsilon\rangle$ from LITOS to $\varepsilon$ inferred from the Thorpe analysis indicated by the ratio $\left\langle\varepsilon_{\text {LITOS }}\right\rangle / \varepsilon_{\text {Thorpe }}$ of the blue and green curves in Fig. 7 is plotted in Fig. 8. It reveals a large range of 5 orders of magnitude. Overall, for $71 \%$ (BEXUS 8: 64\%) of the layers, $\varepsilon$ inferred from the Thorpe analysis is larger than the value from the spectral analysis. That the ratio is sometimes larger and sometimes smaller than unity illustrates that the most likely value of $c^{2}$ does not contain the whole information, but the width of the distribution is important. The correlation coefficient between $\left\langle\varepsilon_{\text {LITOS }}\right\rangle$ and $\varepsilon_{\text {Thorpe }}$ is 0.06 (BEXUS 8: 0.39). Due to the influence of the Brunt-Väisälä frequency, pronounced peaks in $L_{\mathrm{O}}$ or $L_{\mathrm{T}}$ (Fig. 3) do not necessarily correspond to large $\varepsilon$ (Fig. 7), e.g. at $\sim 5 \mathrm{~km}$.

\section{Discussion and conclusions}

In this paper, the first extensive examination of the relation between the Thorpe length $L_{\mathrm{T}}$ and Ozmidov scale $L_{\mathrm{O}}$ for stratospheric conditions was performed, using the new high-resolution instrument LITOS and a radiosonde on the same gondola. Therewith, the assumption for computing energy dissipation rates $\varepsilon$ from a Thorpe analysis of stan- 
dard radiosondes, namely the proportionality $L_{\mathrm{O}} \propto L_{\mathrm{T}}$, was checked. In our data no obvious relation between $\overline{L_{\mathrm{O}}}$ and $L_{\mathrm{T}}$ can be seen, particularly no proportionality. The proportionality "constant" used in radiosonde analyses, $c^{2}=$ $\left(\overline{L_{\mathrm{O}}} / L_{\mathrm{T}}\right)^{2}$, shows a very broad distribution with a width of $\sim 2$ orders of magnitude. This is also reflected in the large deviation of $\varepsilon$ values up to a factor of $\sim 3000$ obtained with both methods. Nevertheless, although the values for individual layers are highly variable, the mean of $c^{2}$ is 0.1 for both BEXUS 12 and BEXUS 8, which is close to 0.3 used by Clayson and Kantha (2008), who reviewed oceanic measurements to obtain that value. Kantha and Hocking (2011) obtained $c^{2}=1.0$ by a comparison of radiosonde data to radar measurements. Gavrilov et al. (2005) used $c^{2}=1.32(c=$ 1.15) referring to a French thesis; this value was obtained from selected thick stratospheric layers $(>200 \mathrm{~m})$ with statistically homogeneous turbulence. However, in those publications no data basis, distribution width or error is given. Recently, Wilson et al. (2014) reported a few case studies of turbulent layers in the troposphere detected simultaneously by radar and balloon; using their reported estimates of $L_{\mathrm{T}}$ and $L_{\mathrm{O}}$ leads to values of $c^{2}$ between 0.1 and 1.6.

One reason for discrepancies from the proportionality $L_{\mathrm{O}} \propto L_{\mathrm{T}}$ may be that large overturns might change significantly during the time the sensor needs to fly through the layer, such that the sorting procedure then no longer makes sense. Furthermore, direct numerical simulations by Smyth and Moum (2000) indicate that $L_{\mathrm{O}} / L_{\mathrm{T}}$ is not constant but rather depends on the age of turbulence.

Some turbulent layers are not detected at all by the Thorpe analysis. Those are not associated with a (significant) negative gradient of potential temperature, which is necessary for detection by the Thorpe method. Not all turbulence is related to static instabilities. Even if initially a negative potential temperature gradient may have occurred, it is removed by the turbulent motions which outlive the instability; such fossil turbulence cannot be detected by the Thorpe method. Apart from that, turbulent layers may be too thin to be observed with the relatively coarse vertical resolution of the radiosonde. On the other hand, some unstable layers detected by the Thorpe analysis are not observed by LITOS. An explanation is that the static instability may not yet have led to turbulent motions. In these cases, a correspondence between both measurements is not expected.

Not all layers are detected by both systems. Of the significant layers detected by the Thorpe analysis, $86 \%$ (BEXUS 8) and $69 \%$ (BEXUS 12) are also detected by LITOS. For BEXUS 12, the mean thickness of significant, unstable layers as detected by the Thorpe analysis is $53 \mathrm{~m}$. The mean thickness of those significant layers also detected by LITOS is $63 \mathrm{~m}$; that of significant layers not detected by LITOS is only $31 \mathrm{~m}$. That means that the simultaneous detection depends on the size of the layer; mainly thin layers are detected by only one method. But as this only applies to $\lesssim 30 \%$ of the layers, and those layers were taken out of the comparison, the bias for our results should be small.

For LITOS, the detection limit for $l_{0}$ on small scales (i.e. high frequencies or large $\varepsilon$ ) is given by the sampling rate. This limit has been encountered in a few cases for small regions where the inertial range extends further than the Nyquist limit of $4 \mathrm{kHz}$. For large scales (i.e. low frequencies or small $\varepsilon$ ), the detection limit is determined by the trend removal and the window length. As a reasonable part of the inertial range has to be resolved to enable a fit, the limit is estimated to $\sim 1 \mathrm{~m}$. The maximal identified $l_{0}$ values of $10 \mathrm{~cm}$ and $8.7 \mathrm{~cm}$ for BEXUS 8 and BEXUS 12, respectively, were far below this limit. That means that these limitations do not affect the results.

To date, we have only two flights with data usable for the analysis presented in this paper, namely BEXUS 8 and BEXUS 12, which both took place at polar latitudes near autumn equinox. Of course they cannot represent the whole variability of the stratosphere. Nevertheless, although there are differences between both flights, such as dissipation rates being on average 1 order of magnitude higher for BEXUS 8 , these are not relevant for the results discussed above. More flights with our new high-resolution instrument are planned to broaden the data basis.

Our results question the applicability of the Thorpe analysis for the extraction of energy dissipation rates for individual turbulent layers. Nevertheless, statements in the statistical mean seem to be possible. Further research on the relation between Thorpe and Ozmidov lengths and the temporal evolution of turbulence is necessary.

Acknowledgements. The data from the BEXUS 8 flight were kindly provided by Anne Haack. The BEXUS programme was financed by the German Aerospace Center (DLR) and the Swedish National Space Board (SNSB). We are grateful for the support by the "International Leibniz Graduate School for Gravity Waves and Turbulence in the Atmosphere and Ocean" (ILWAO) funded by the Leibniz Association (WGL). Furthermore, we would like to thank Lars Umlauf for helpful discussions. We thank the reviewers Richard Wilson and Marvin Geller for their helpful comments.

Edited by: W. Ward 


\section{References}

Barat, J.: Some characteristics of clear-air turbulence in the middle stratosphere, J. Atmos. Sci., 39, 2553-2564, doi:10.1175/15200469(1982)039<2553:SCOCAT>2.0.CO;2, 1982.

Clayson, C. A. and Kantha, L.: On turbulence and mixing in the free atmosphere inferred from high-resolution soundings, J. Atmos. Ocean. Tech., 25, 833-852, doi:10.1175/2007JTECHA992.1, 2008.

Dillon, T. M.: Vertical overturns: A comparison of Thorpe and Ozmidov length scales, J. Geophys. Res., 87, 9601-9613, 1982.

Gavrilov, N. M., Luce, H., Crochet, M., Dalaudier, F., and Fukao, S.: Turbulence parameter estimations from high-resolution balloon temperature measurements of the MUTSI-2000 campaign, Ann. Geophys., 23, 2401-2413, doi:10.5194/angeo-23-24012005, 2005.

Haack, A., Gerding, M., and Lübken, F.-J.: Characteristics of stratospheric turbulent layers measured by LITOS and their relation to the Richardson number, J. Geophys. Res., 119, 10605-10618, doi:10.1002/2013JD021008, 2014.

Heisenberg, W.: Zur statistischen Theorie der Turbulenz, Z. Phys., 124, 628-657, doi:10.1007/BF01668899, 1948.

Kantha, L. and Hocking, W. K.: Dissipation rates of turbulence kinetic energy in the free atmosphere: MST radar and radiosondes, J. Atmos Sol.-Terr. Phy., 73, 1043-1051, doi:10.1016/j.jastp.2010.11.024, 2011.

Love, P. T. and Geller, M. A.: Research using high (and higher) resolution radiosonde data, T. Am. Geophys. Un., 93, 337-338, doi:10.1029/2012EO350001, 2012.

Lübken, F.-J.: On the extraction of turbulent parameters from atmospheric density fluctuations, J. Geophys. Res., 97, 20385-20395, doi:10.1029/92JD01916, 1992.

Luce, H., Fukao, S., Dalaudier, F., and Crochet, M.: Strong Mixing Events Observed near the Tropopause with the MU Radar and High-Resolution Balloon Techniques, J. Atmos. Sci., 59, 2885-2896, doi:10.1175/15200469(2002)059<2885:SMEONT>2.0.CO;2, 2002.

Ozmidov, R. V.: On the turbulent exchange in a stably stratified ocean, Izv. AN. Fiz. Atmos. Ok., 1, 853-860, translated from Russian by Danielle and Victor Barcilon, 1965.
Smyth, W. D. and Moum, J. N.: Length scales of turbulence in stably stratified mixing layers, Phys. Fluids, 12, 1327-1342, doi:10.1063/1.870385, 2000.

Theuerkauf, A., Gerding, M., and Lübken, F.-J.: LITOS - a new balloon-borne instrument for fine-scale turbulence soundings in the stratosphere, Atmos. Meas. Tech., 4, 55-66, doi:10.5194/amt-4-55-2011, 2011.

Thorpe, S. A.: Turbulence and mixing in a Scottish loch, Philos. Tr. R. Soc. S-A, 286, 125-181, doi:10.1098/rsta.1977.0112, 1977.

Thorpe, S. A.: The Turbulent Ocean, Cambridge University Press, Cambridge, section 6.3.2, 2005.

Wesson, J. C. and Gregg, M. C.: Mixing at Camarinal Sill in the Strait of Gibraltar, J. Geophys. Res., 99, 9847-9878, doi:10.1029/94JC00256, 1994.

Wijesekera, H. W., Dillon, T. M., and Padman, L.: Some statistical and dynamical properties of turbulence in the oceanic pycnocline, J. Geophys. Res., 98, 22665-22679, doi:10.1029/93JC02352, 1993.

Wilson, R., Luce, H., Dalaudier, F., and Lefrère, J.: Turbulence patch identification in potential density or temperature profiles, J. Atmos. Ocean. Tech., 27, 977-993, doi:10.1175/2010JTECHA1357.1, 2010.

Wilson, R., Dalaudier, F., and Luce, H.: Can one detect small-scale turbulence from standard meteorological radiosondes?, Atmos. Meas. Tech., 4, 795-804, doi:10.5194/amt-4-795-2011, 2011.

Wilson, R., Luce, H., Hashiguchi, H., Shiotani, M., and Dalaudier, F.: On the effect of moisture on the detection of tropospheric turbulence from in situ measurements, Atmos. Meas. Tech., 6, 697702, doi:10.5194/amt-6-697-2013, 2013.

Wilson, R., Luce, H., Hashiguchi, H., Nishi, N., and Yabuki, Y.: Energetics of persistent turbulent layers underneath mid-level clouds estimated from concurrent radar and radiosonde data, J. Atmos. Sol.-Terr. Phy., 118, Part A, 78-89, doi:10.1016/j.jastp.2014.01.005, 2014. 